\title{
Understanding Protein Glycosylation Pathways in Bacteria
}

\begin{abstract}
Through advances in analytical methods to detect glycoproteins and to determine glycan structures, there have been increasing reports of protein glycosylation in bacteria. In this review, we summarize the known pathways for bacterial protein glycosylation: 1) lipid carrier-mediated "en bloc" glycosylation; and 2) cytoplasmic stepwise protein glycosylation. The exploitation of bacterial protein glycosylation systems, especially the "mix and match" of three independent but similar pathways (oligosaccharyltransferase-(OST)-mediated protein glycosylation, lipopolysaccharide (LPS) and peptidoglycan biosynthesis) in Gram-negative bacteria for glycoengineering recombinant glycoproteins is also discussed.
\end{abstract}

\author{
Abbreviations: \\ Gal, galactose; Glc, glucose; GlcNAc, $N$-acetylglucosamine; \\ diNAcBac, 2,4-diacetamido-2,4,6-trideoxy- $\alpha$-D-glucopyranose; \\ GalNAc, $N$-acetylgalacosamine; UndP, undecaprenyl pyrophosphate; \\ Hex, hexose; FucNAc, $N$-acetylfucosamine; \\ Xyl, xylose; DATDH, 2,4-diacetamido-2,4,6-trideoxyhexose; \\ GATDH, 2-glyceramido-4-acetamido-2,4,6-trideoxyhexose; \\ $\alpha-5 \mathrm{~N} \beta \mathrm{OHC} 47 \mathrm{NFmPse}, 5-N$ - $\beta$-hydroxybutyryl-7- $N$-formyl-pseudaminic acid; \\ Man, mannose; PP, polyprenol phosphate. Pse, pseudaminic acid; \\ Leg, 5,7-diacetamido-3,5,7,9-tetradeoxy-D-glycero-D-galacto-nonulosonic acid.
}

\section{Key words}

Protein glycosylation, glycosylation pathway, glycoengineering 


\section{Introduction}

Bacteria have evolved complex biosynthetic pathways to generate an array of monoor oligosaccharide moieties to produce capsules, lipopolysaccharides (LPS), peptidoglycans, and also to glycosylate lipids (including membrane cholesterols) and proteins. Glycosylation requires the action of enzymes known as glycosyltransferases (GTs) to recognize both the activated-sugar donor and the corresponding acceptor to form a new glycosidic bond between the sugar and the acceptor. The donors are phosphate-activated sugars, which can be linked to nucleotides (UDP-, GDP-, ADPand CMP-) or dolichol-related lipid groups.

Protein glycosylation is a complex enzymatic process that involves GTs and activated substrates. Once thought to be exclusively present in eukaryotes, it is now evident that protein glycosylation is a common feature in all three domains of life (Eukarya, Bacteria, and Archaea) [1, 2]. Even though the mass content of glycans in a glycoprotein is often small (5-10\%), glycans represent a significant part of the hydrodynamic volume of the overall glycoprotein, as glycans have a much lower density than proteins [3]. Glycans can have a profound influence on the physiochemical properties of a protein such as proper folding, aggregation, solubility, and stability $[4,5]$. Recent studies have shown that glycans on bacterial proteins play key functions in biological processes including adhesion, immune evasion and host colonization $[6,7]$. In this review, we summarize the well-described bacterial protein glycosylation pathways, and discuss its exploitation for glycoengineering.

\section{An Overview of Bacterial Protein Glycosylation}

The first confirmed report of $N$-linked glycosylation pathway was described in 2002 in the human gastrointestinal pathogen Campylobacter jejuni [8]. Since then a large number of bacterial glycoproteins have been identified including 1) the flagellins from C. jejuni [9, 10], Campylobacter coli [11], Helicobacter pylori [12-14], Aeromonas caviae [15], Pseudomonas aeruginosa [16], Listeria monocytogenes [17, 
18], Clostridium difficile [19]; 2) the pilins from Neisseria meningitidis [20], Neisseria gonorrhoeae [21], and P. aeruginosa [22], and 3) the heptosylated autotransporters including AIDA-I, TibA and Ag43 from three different pathogenic $E$. coli strains [23-26] and CARC from Citrobacter rodentium [6]. In addition, a number of proteins from Bacteroides fragilis [27], the Fap1 (fimbriae-associated protein 1) from an oral bacterium Streptococcus parasanguinis [28] and the adhesins HMW1/HMW2 from the non-typeable Haemophilus influenzae are also glycoproteins $[29,30]$. O-linked glycoproteins have also been reported in Porphyromonas gingivalis [31, 32], Burkholderia [33, 34], Francisella [35, 36] and Acinetobacter spp.[37, 38].

The glycans of bacterial glycoproteins are more complex than their eukaryotic counterparts [5], and carbohydrates including diNAcBac [39], pseudaminic acid (Pse) [40], DATDH and GATDH [41] are found only in bacteria. Mass spectrometry and NMR analysis are commonly applied analytical tools to solve the precise structure of these glycans, however, owing to the requirement of large amounts of highly purified materials and the high level of specialization to interpret the data [42], the structures of many bacterial protein glycans are not fully characterized.

Based on the glycosidic linkage, protein glycosylation can be categorized into two major types: 1) $O$-glycosylation, in which the glycans are attached to the hydroxyl oxygen of serine or threonine (Ser/Thr) residues and 2) $\mathrm{N}$-glycosylation, in which the glycans are attached to the amide nitrogen of asparagine (Asn) residues in targeted proteins. In eukaryotes and archaea, the Asn residues are present in the tripeptide consensus sequence: Asn-X-Ser/Thr (X can be any amino acid except proline), whereas in bacteria this consensus sequon is extended to be Asp/Glc-X1-Asn- X2-Ser/Thr (X1 and X2 represent any amino acid except proline) [43].

Depending on how the mono- or oligosaccharides are transferred to the targeted proteins, protein glycosylation can also be categorized into two main pathways: 1) lipid carrier-mediated "en bloc" glycosylation and; 2) stepwise protein glycosylation. 
En bloc glycosylation involves the preassembly of the glycan onto a lipid carrier to form a lipid-linked oligosaccharide (LLO), which is then transported by a flippase from the cytoplasm to the periplasm, where the oligosaccharide is transferred as whole from the LLO to target proteins by an oligosaccharyltransferase (OST) [1]. In Gram-negative bacteria, the glycans are first preassembled onto an undecaprenyl pyrophosphate (UndP), while in the Gram-positive bacteria Actinomycetes, protein $O$-mannosylation utilizes polyprenol phosphate (PP) as the lipid carrier [44]. Stepwise protein glycosylation involves the sequential addition of nucleotide-activated sugars onto Asn and Ser/Thr residues of targeted proteins by GTs.

\section{Lipid Carrier-Mediated en bloc Protein Glycosylation}

Three different categories of lipid carrier-mediated en bloc protein glycosylation in bacteria are described here: 1) the OST-mediated protein $N$-glycosylation in Gram-negative bacteria; 2) the OST-mediated protein $O$-glycosylation in Gram-negative bacteria and; 3) the $O$-mannosylation in Gram-positive Actinomycetes (Figure 1).

\subsection{OST-Mediated Protein $\mathrm{N}$-glycosylation in Campylobacter and Helicobacter} spp. The protein $N$-glycosylation pathway in $C$. jejuni (Figure 1A) is the best-studied glycosylation system in bacteria and has been considered as a prototype for studying and appreciating the importance of protein glycosylation in host-microbe interactions. This well-characterized system is encoded by a unique $17-\mathrm{kb} N$-linked protein glycosylation locus that contains 12 open reading frames (ORF). The enzymes encoded within this locus are responsible for glycosylating more than 60 different proteins with the heptasaccharide, GalNAc-GalNAc-Glc-GalNAc-GalNAc-GalNAc-diNAcBac [39]. The sugar donor for diNAcBac in Campylobacter is UDP-diNAcBac, which is synthesized from the universal substrate UDP-GlcNAc (Figure 2) [2, 5, 45, 46]. The assembly of the heptasaccharide onto the lipid carrier involves five GTs: the first GT PglC (Cj1124c) 
is responsible for the transfer of diNAcBac to the lipid carrier UndP, forming UndP-linked diNAcBac [47]; the second GT PglA (Cj1125c) adds the second GalNAc residue [48]; Subsequently, the third GT PglJ (Cj1127c) adds the third GalNAc residue [48]; the fourth GT PglH $(\mathrm{Cj} 1129 \mathrm{c})$ then acts as a polymerase to transfer another three GalNAc residues to form a UndP-linked hexasaccharide [49]; finally, the fifth GT PgII (Cj1128c) adds the branching Glc residue to complete the assembly of lipid-linked heptasaccharide [50]. After the assembly in the cytoplasm, the LLO is then translocated by the flippase PgIK $(\mathrm{Cj} 1130 \mathrm{c})$ into the periplasm, where the LLO is transferred en bloc by the OST PglB (Cj1126c) to acceptor proteins [39] (Figure 1A).

Like C. jejuni, all the other Campylobacter species possess a similar $N$-glycosylation gene cluster and therefore can produce a range of structurally distinct $N$-linked glycans with variations in the number of sugar residues (penta-, hexa-, and heptasaccharides) in the presence of branching sugars and in the overall monosaccharide content [51].

There are several other bacterial genera, including Helicobacter and Wolinella, that belong to the same phylogenetic grouping as Campylobacter, the $\varepsilon$-proteobacteria [1], and therefore it is not surprising that similar $N$-glycosylation pathways have also been confirmed in Helicobacter [52] and Wolinella spp.[1, 51]. Three closely related Helicobacter species (H. pullorum, H. canadenis and H. winghamensis) contain two unrelated $p g l B$ genes $(p g l B 1$ and pglB2) [52]. It has been shown in H. pullorum that PglB1 is the OST responsible for the $N$-linked protein glycosylation with a pentasaccharide glycan comprised of two HexNAc residues and three unknown sugar residues with MWs of $217 \mathrm{Da} 217 \mathrm{Da}$ and $216 \mathrm{Da}$ [52]. Of note is that except for the above three Helicobacter species, most other Helicobacter species, including $H$. pylori, lack the $p g l B$ gene [5], and thus it is very likely that $N$-linked protein glycosylation is absent in these bacteria. The $N$-glycosylation pathway found in Wolinella succinogenes has been shown to produce a hexasaccharide comprising one Hex residue, one DATDH and four unknown sugar residues with MWs of 216 Da, 
$216 \mathrm{Da}, 216 \mathrm{Da}$ and $232 \mathrm{Da}[1,51]$.

In addition to the $\varepsilon$-proteobacteria mentioned above, Desulfovibrio vulgaris and Desulfovibrio desulfuricans, which belong to $\delta$-proteobacteria, have also been found to possess an $N$-linked protein glycosylation pathway [2].

\subsection{OST-Mediated Protein $O$-Glycosylation in Gram-Negative Bacteria}

Similar to the general protein $\mathrm{N}$-glycosylation system in C. jejuni, several bacteria including N. meningitidis [20] and N. gonorrhoeae [21] (Figure 1B), P. aeruginosa (Figure 1C), Acinetobacter baumannii [37] (Figure 1D), and also B. fragilis [27] and Francisella tularensis [36] are found to possess a general $O$-linked glycosylation system which encodes enzymes including GTs, flippases and OSTs required for the assembly, translocation and ligation of oligosaccharides to target proteins.

In N. meningitidis, pilin glycosylation genes ( $p g l B$ or $p g l B 2, p g l C, p g l D, p g l A, p g l E$ and $p g l F$ ) are responsible for the glycosylation of the major subunit of type IV pili PilE and the surface-exposed nitrite reductase AniA with a trisaccharide composed of Gal-Gal-DATDH/GATDH) [41]. Of note, is that the structure of DATDH was later unequivocally shown to be diNAcBac [53]. The sugar donor for diNAcBac/GATDH in $N$. meningitidis is UDP-diNAcBac/GATDH, which is synthesized from UDP-GlcNAc [2] (Figure 3). The first step is catalyzed by PgID (homologous to the C. jejuni PglF) to mediate the dehydration of UDP-GlcNAc; PglC (homologous to the C. jejuni PglE) in the second step transfers the amino group; the third step is then catalyzed by the acetyltransferase function of PglB or PglB2 to form UDP-DATDH or UDP-GATDH, respectively. Subsequently, the bifunctional enzyme PglB or PglB2 utilizes its GT function to transfer the activated diNAcBac or GATDH to the lipid carrier. The Gal transferases PglA and PglE are responsible for the transfer of Gal residues to the diNAcBac or GATDH to form a lipid-linked trisaccharide [54]. The flippase PglF then translocates the lipid-linked trisaccharide to the periplasm, where PglL (OST) transfers the oligosaccharide to glycosylate PilE or AniA [55] (Figure 
1B). Very recently, it was shown that the PilE of class II pilins in N. meningitidis is heavily coated with glycans, having up to five glycosylation sites, thus limiting the access of antibodies to the pilin polypeptide chain and mediating immune evasion from the host [56]. This is an excellent example demonstrating the importance of protein glycosylation in bacterial pathogenesis.

Compared to only two glycoproteins PilE and AniA in N. meningitidis, eleven membrane-associated proteins have been demonstrated to be glycosylated in $N$. gonorrhoeae [21]. Homologous to PglL in N. meningitidis, $\mathrm{PglO}$ in N. gonorrhoeae is the OST responsible for the transfer of glycans to proteins [21] (Figure 1B). Both PglO and PglL contain the signature Wzy_C motif present in LPS $O$-antigen ligase WaaL, which transfers an undecaprenyl-linked $O$-antigen to the LPS lipid A-core [57]. Interestingly, the OST PilO (contains the Wzy_C motif) in P. aeruginosa 1244 transfers a single $O$-antigen unit, a trisaccharide comprised of 5NßOHC47NFmPse-Xyl-FucNAc, generated from the LPS $O$-antigen biosynthetic pathway to glycosylate the pilin subunit PilA $[58,59]$ (Figure 1C). WbpL initiates the assembly of the single $O$-antigen unit by the transfer of FucNAc from UDP-FucNAc to UndP carrier $[60,61]$. The GTs for the transfer of $\mathrm{Xyl}$ and $5 \mathrm{~N} \beta \mathrm{OHC} 47 \mathrm{NFmPse}$ are unknown and once assembly of the $O$-antigen unit is complete, it is translocated by the $O$-antigen flippase Wzx to the periplasm, where it is used by the $O$-antigen ligase WaaL for the ligation of $O$-antigen with the LPS lipid A-core, and it is also used by the OST PilO for the glycosylation of PilA [22], suggesting an evolutionary collection between LPS biosynthesis and OST-mediated pilin glycosylation in Gram-negative bacteria.

Very recently, the general $O$-linked glycosylation system has been identified and characterized in the opportunistic nosocomial pathogen A. baumannii [37, 62] (Figure 1D). Multiple proteins in A. baumannii strain ATCC 17978 were found be modified with a branched pentasaccharide comprising of GalNAc, Glc, Gal, GlcNAc and a glucuronic acid derivative, GlcNAc3NAcA4OAc [37, 62]. Interestingly, this 
pentasaccharide also serves as the building block for capsular polysaccharides, suggesting a common pathway for $O$-linked protein glycosylation and capsule biosynthesis in A. baumannii [62]. As this common pathway is dependent on the activity of PglC (the initiating enzyme for the synthesis of the pentasaccharide), it has been proposed that the assembly of the pentasaccharide is initiated by the transfer of GalNAc to the UndP carrier, by PglC. Four GTs (A1S_0058, A1S_3482, A1S_0059, and A1S_0060) transfer corresponding sugars to complete the pentasaccharide. Wzx (A1S_0056) flips the completed pentasaccharide structure to the periplasm and subsequently coupled to target proteins by the OST PgIL (A1S_3176)[62] (Figure 1D).

Other examples of general OST-mediated protein $O$-glycosylation systems are found in Bacteroides [27] and F. tularensis [36]. Bacteroides comprise one of the most abundant genera of commensals in the human colon, and it has been shown that $O$-glycosylation is central not only to the physiology of $B$. fragilis but is also required for competitive colonization of its mammalian host [27]. The $O$-glycan in $B$. fragilis has been demonstrated to be an oligosaccharide comprising of nine sugar units, three Hex residues (one reduced), two hexuronic acids, two HexNAc residues, one methylated deoxyhexose and one deoxyhexose residue [63]. Five putative GTs (BF4299, BF4300, BF4301, BF4305, BF4306) and a fucosyltransferase are proposed to be involved in the assembly of the glycan, and the $O$-antigen flippase Wzx (BF4298) is thought to be responsible for the translocation of lipid-linked glycan from the cytoplasm to the periplasm [27]. However, the OST in B. fragilis has yet to be identified.

Pilin protein PilA (encoded by FTS_0381) [36] and a lipoprotein encoded by gene FTS_0065 [35] in F. tularensis FSC200 strain, and a virulence factor DsbA protein in F. tularensis SchuS4 strain [64], were found to be glycosylated with a phosphate-containing hexasaccharide (HexNAc-unknown sugar-HexNAc-Hex-Hex-HexNAc). The OST involved in the en bloc glycosylation 
was identified as PglA (encoded by FTL_0425 in FCS200 strain, and FTT_0905 in SchuS4 strain) [36]. The $12.5 \mathrm{kB}$ gene cluster FTT0789-FTT0800 in the SchuS4 strain was shown to be involved in the assembly and translocation of the hexasaccharide [64, 65]. Interestingly, the mutation of $w b t C$ gene from LPS biosynthetic locus resulted in not only altered LPS but also reduced protein glycosylation [66]. Similarly the mutation of the putative glycan flippase gene FTS_1402 in the FCS200 strain lead to alteration in protein glycosylation and also a decrease in LPS production [65], suggesting a cross-talk between LPS biosynthesis and protein glycosylation in $F$. tularensis.

\subsection{Protein $O$-Mannosylation in Gram-Positive Actinomycetes}

A general protein $O$-mannosylation pathway has been found in Gram-positive bacteria Actinomycetes, which includes the genera of Mycobacterium [67, 68], Corynebacterium [69], and Streptomyces [70]. It has been shown that the membrane-bound phosphate binding protein PstS in Streptomyces coelicolor is glycosylated with a Man-linked trihexose [70]. The polyprenylphosphate Man synthase Ppm1 is known to be responsible for catalyzing the transfer of Man from GDP-Man to the lipid carrier polyprenyl phosphate, forming polyprenyl monophosphomannose [70]. However, how the lipid-linked Man is further extended to the trihexose moiety is not clear. The $O$-mannosyltransferase Pmt catalyzes the glycosylation of the Ser or Thr residues of PstS with the Man-linked trihexose [2, 70].

\section{Stepwise Protein Glycosylation}

As mentioned earlier, bacterial stepwise protein glycosylation is OST-independent and is catalysed by GTs, which sequentially transfer monosaccharides directly to an acceptor protein. Some representative examples of stepwise protein glycosylation include the $N$-glycosylation of adhesin protein HMW1 in H. influenzae [29, 30], the $O$-glycosylation of flagellins in Campylobacter [40] and Helicobacter [12, 13], and also the heptosylation of autotransporters [6]. 


\subsection{Stepwise Protein $\mathrm{N}$-glycosylation in $\mathrm{H}$. influenzae.}

$N$-linked glycosylation of the $H$. influenzae HMW1 adhesion occurs in the cytoplasm and involves the direct transfer of Hex or Hex-Hex residues to 31 sites on the HMW1 protein $[29,30]$ (Figure 4A). The Hex is either Gal or Glc, and all but one of the glycosylation sites are Asn residues within the eukaryotic-like sequon Asn-X-Ser/Thr $[29,30]$. The GT responsible for this process has been confirmed as HMW1C, which has been assigned as a member of CAZy (Carbohydrate Active Enzyme database) family GT41 [30, 71, 72]. Interestingly, homology analysis reveals 42-68\% identity and $58-83 \%$ similarity between the full-length HMW1C sequence and proteins in a number of other Gram-negative bacteria, suggesting a GT41 family of bacterial HMW1C-like proteins with $N$-glycosylation glycosyltransferase activity [30]. Indeed, the HMW1C-like protein in Actinobacillus pleuropneumoniae has been shown to be able to transfer Gal or Glc to known Asn glycosylation sites in HMW1 [73].

\subsection{Stepwise Protein $O$-Glycosylation of Bacterial Flagellins.}

Bacterial flagellins are the most well studied proteins with stepwise cytoplasmic $O$-glycosylation. Flagellins from $C$. jejuni and C. coli [40], H. pylori $[12,13]$, and $A$. caviae [15] are modified with the 9-carbon sialic acid-like sugar Pse or legionaminic acid (Leg) and it has been demonstrated that flagellin glycosylation is essential for motility and flagella assembly in these bacteria $[74,75]$. In contrast, the glycosylation of flagellin structural protein FliC in $P$. aeruginosa is not required for flagellar motility and assembly. FliC in P. aeruginosa PAK is modified with a heterogeneous $O$-linked glycan comprising up to 11 additional monosaccharides, linked to the protein backbone via a rhamnose residue [16].

C. jejuni strain 81-176 flagellins have been shown to be predominantly modified with Pse5Ac7Ac with additional heterogenous derivatives, including Pse5Am7Ac (Figure 4B). This imparts an approximate $6 \mathrm{kDa}$ shift in the molecular mass of the predicted protein $[40,75]$. Similarly for $C$. coli VC 167 T2, the flagellins are extensively 
modified with Pse5Ac7Ac and its derivatives [40]. In addition to Pse, the flagellins of C. jejuni 11168 and C. coli VC167 are also found to be glycosylated with Leg and its derivatives [76].

Compared to the Campylobacter flagellin, which are glycosylated with numerous Pse/Leg and related derivatives, the glycosylation of $H$. pylori flagellin is far simpler, and only a single sugar species (Pse5Ac7Ac) is present on H. pylori flagellin [40]. It has been demonstrated that H. pylori strain 1061 flagellin are glycosylated with Pse5Ac7Ac at 7 sites in FlaA and 10 sites in FlaB [13] (Figure 4C).

A common feature of flagellin glycosylation is that the sites of glycosylation are located in the central, surface-exposed domain of flagellin when it is assembled in a filament, and the mechanism of attachment seems to be unrelated to a consensus peptide sequence, but is rather related to the surface accessibility of the Ser/Thr residues in the folded protein $[13,16,40]$.

The sugar donor for Pse in H. pylori and C. jejuni has been shown to be CMP-Pse [13]. Six Pse biosynthesis enzymes (PseB, C, H, G, I, F) have been found to constitute the complete CMP-Pse biosynthetic pathway starting from UDP-GlcNAc [14] (Figure 5).

The disruption of any of the Pse genes in this pathway results in a non-motile phenotype, no structural flagella filament and severely reduced levels of flagellin protein $[13,45,77,78]$. The absence of glycosyated flagellins in the cytoplasm of the H. pylori wild-type or pse mutants suggests that flagellin glycosylation might be coupled with their secretion by the flagella Type III secretion system [77].The sugar donor for Leg in Campylobacter is CMP-Leg, and eleven enzymes have been successfully reconstituted in vitro for the biosynthesis of CMP-Leg starting from Fru-6P [79] (Figure 6). 
Although the biosynthesis of CMP-Pse and CMP-Leg have been elucidated, the key GTs involved in the transfer of Pse/Leg and related derivatives to flagella are yet to be identified and confirmed. In H. pylori, HP0114 is not directly involved in the biosynthesis of CMP-Pse, but its inactivation resulted in a non-motile phenotype, and therefore, it has been suggested that HP0114 might encode the pseudaminyltransferase [13]. A recent study showed that the motility-associated factor gene mafl in A. caviae might be the putative pseudaminyltransferase responsible glycosylation of the flagellin in this microbe [15].

Flagellin $O$-glycosylation has also been demonstrated in the Gram-positive bacteria $L$. monocytogenes [17], Clostridium botulinum [80] and C. difficile [19]. Up to six sites of the central surface-exposed region of the flagellin monomer of L. monocytogenes is glycosylated with $\beta$-O-linked GlcNAc, which is transferred by the GT protein Lmo0688 $[12,17]$. The flagellins of $C$. botulinum and $C$. difficile have been shown to be modified with Leg derivatives and HexNAc residues, respectively $[19,80]$.

\subsection{Stepwise Heptosylation of Autotransporters}

Autotransporters constitute the largest number of secreted virulence factors in Gram-negative bacteria. The modular structure of autotransporters consist of a signal peptide, an $N$-terminal functional passenger domain, and a $C$-terminal $\beta$-barrel domain which mediates the translocation of the passenger domain across the bacterial outer membrane $[6,81]$. The passenger domains of autotransporters TibA from enterotoxigenic E. coli (ETEC) strain H10407 [82, 83], AIDA-I from diarrheagenic E. coli (DAEC) clinical isolate 2787 [26, 82], and Ag43 from pathogenic as well as commensal E. coli strains [23, 84] have long been known to be extensively glycosylated with $O$-linked Hep moieties. Very recently, it has been demonstrated that heptosylation of AIDA-I is catalyzed by the heptosyltransferase AAH and its paralog AAH2, and this modification is essential for adhesion to host cells [6]. Structural 
analysis of the heptosylated TibA passenger domain revealed that TibA was modified by the heptosyltransferase TibC with 35 Hep moieties, which are exclusively on Ser residues [6].

AAH/AAH2 and TibC are members of the newly-defined bacterial autotransporter heptosyltransferase (BAHT) family that contain a ferric ion and adopt a dodecamer assembly [6]. The gene encoding BAHT is always located upstream of its substrate autotransporter in the same operon [6] (Figure 7A). CARC, an AIDA-like autotransporter from $C$. rodentium is hyper-heptosylated by its cognate heptosyltransferase BAHTCr (Figure 7A), and this modification is essential for colonization in mice [6]. In addition, BAHTs have also been identified in Salmonella enterica serovar Urbana R8-2977, Shigella sp. D9, Laribacter hongkongensis HLHK9, Cronobacter sakazakii ATCC BAA-894, and Burkholderia spp., and these BAHT proteins share $40 \%-80 \%$ homology to AAH [6].

The sugar donors utilized by BATH for the heptosylation of autotransporters are recruited from the LPS biosynthetic pathway, in which ADP-DD-Hep and ADP-LD-Hep are synthesized (Figure 7B). Both ADP-DD-Hep and ADP-LD-Hep could serve as the sugar donors for the glycosylation of autotransporters [6]. The pathway for the stepwise heptosylation of autotransporters by BAHT is summarized in (Figure 7C).

\section{The "Mix and Match" of Three Independent Glycosylation Pathways for Glycoengineering}

In Gram-negative bacteria, the biosynthetic pathways of OST-mediated protein glycosylation, LPS and peptidoglycan synthesis are independent, but all follow a similar route: 1) using UndP as a lipid carrier to form LLO; 2) the translocation of LLO to the periplasm by flippases and; 3) the ligation of glycans to acceptors by glycosyltransferases. An evolutionary connection between LPS synthesis and 
OST-mediated protein glycosylation has been suggested [57, 85]. For example, the OST-mediated $N$-glycosylation flippase PglK in $C$. jejuni shares $37 \%$ identity with the LPS $O$-antigen flippase Wzk in $H$. pylori, and both enzymes are functionally exchangeable [85]. More importantly, both LPS $O$-antigen ligases (WaaL) and $O$-OSTs contain the periplasmic domain Wzy_C motifs, however based on current knowledge it is impossible to precisely differentiate based on protein sequences alone whether a Wzy_C motif containing peptide functions as a ligase or O-OST [57]. Remarkably, the lipid-linked $O$-antigen 5N $3 O H C 47 N F m P s e-X y l-F u c N A c$ trisaccharide unit in P. aeruginosa 1244 is utilized in both the LPS and protein glycosylation pathways [20, 57]. In the LPS pathway, the ligase WaaL is responsible for the addition of the $O$-antigen to the lipid A-core, whereas in the protein glycosylation pathway, the OST PilO is responsible for the addition of a single $O$-antigen unit to pilin protein PilA [20, 57].

By taking advantage of the relaxed substrate specificities of the OST, the three independent glycosylation pathways, protein glycosylation, LPS biosynthesis and peptidoglycan biosynthesis, have been "mixed and matched" for glycoengineering a number of recombinant glycoproteins in E. coli and S. enterica [86-90]. It has been shown that $C$. jejuni PglB ( $N$-OST) is capable of transferring various LPS $O$-antigens from E. coli O16, P. aeruginosa O11, and Shigella dysenteriae O1 onto the protein acceptor AcrA in $E$. coli cells $[87,88]$. The commonality among these $O$-antigen oligosaccharides is the $\mathrm{N}$-acetylhexosamine moiety of their reducing sugars (GlcNAc, FucNAc, GalNAc). In contrast, $O$-antigen oligosaccharides with Gal as the reducing sugar could not be transferred by PglB to AcrA [87]. Recently, the X-ray structure of PglB has been resolved and it is thought that the $N$-acetylhexosamine moiety plays a role in the glycosylation mechanism [91]. The relaxed substrate specificities of $O$-OSTs PilO from P. aeruginosa 1244 and PglL from N. meningitidis have also been demonstrated in E. coli $[22,89]$. In contrast to PglB, PglL does not require the 2-acetamido group at the reducing end for activity [74, 89] and so is able to transfer virtually any glycan (including C. jejuni heptasaccharide, E. coli $\mathrm{O} 7$ antigen, E. coli 
K30 capsular structure, $S$. enterica $O$-antigen and E. coli O16 peptidoglycan subunits) to pilin in both E. coli and Salmonella $[22,89]$.

\section{Conclusions and Future Perspectives}

Through advances in genetics, genomics and glycobiology in the past decade, tremendous progress has been made in the field of bacterial protein glycosylation. The identification and elucidation of the complexities of bacterial glycosylation mechanisms have enabled the successful reconstitution of OST-mediated $N$-glycosylation systems of $C$. jejuni, and OST-mediated $O$-glycosylation systems of $P$. aeruginosa and N. meningitidis in E. coli and S. enterica, leading to the production of different recombinant glycoproteins. It is expected that with the ever-increasing repertoire of GTs and OSTs, together with the further understanding and optimization of bacterial glycoengineering systems, the large-scale and cost-effective production of glycoconjugate therapeutics or vaccines in bacteria is not far away from reality. 


\section{Executive Summary}

- Protein glycosylation in bacteria plays key functions in biological processes including adhesion, immune evasion and host colonization.

- Both bacterial protein $\mathrm{N}$-glycosylation and $O$-glycosylation can proceed via lipid carrier-mediated "en bloc" pathway or cytoplasmic stepwise pathway.

- The glycans of bacterial glycoproteins are more complex than their eukaryotic counterparts and many bacterial protein glycans are not fully characterized.

- OST-mediated protein glycosylation, LPS biosynthesis and peptidoglycan biosynthesis pathways (although independent but all proceed via the lipid carrier-mediated "en bloc" pathway), have been "mixed and matched" for glycoengineering a number of recombinant glycoproteins in bacteria. 


\section{References}

$*_{\text {of interest }}$

** of considerable interest

1. Dell A, Galadari A, Sastre F, Hitchen P. Similarities and differences in the glycosylation mechanisms in prokaryotes and eukaryotes. Int $J$ Microbiol. 2010, 148178 (2010).

2. Nothaft H, Szymanski CM. Protein glycosylation in bacteria: sweeter than ever. Nat Rev Microbiol. 8(11), 765-778 (2010). ** Review of both $N$-linked and $\boldsymbol{O}$-linked protein glycosylation pathways in bacteria.

3. Lauc G, Rudan I, Campbell H, Rudd PM. Complex genetic regulation of protein glycosylation. Mol Biosyst. 6(2), 329 (2010).

4. Tian Y, Zhou Y, Elliott S, Aebersold R, Zhang H. Solid-phase extraction of $N$-linked glycopeptides. Nat. Protoc. 2(2), 334-339 (2007).

5. Szymanski CM, Wren BW. Protein glycosylation in bacterial mucosal pathogens. Nat Rev Microbiol. 3(3), 225-237 (2005).

6. Lu Q, Yao Q, Xu Y et al. An iron-containing dodecameric heptosyltransferase family modifies bacterial autotransporters in pathogenesis. Cell Host Microbe. 16(3), 351-363 (2014).

7. Lu Q, Li S, Shao F. Sweet Talk: Protein glycosylation in bacterial interaction with the host. Trends Microbiol. 23(10), 630-641 (2015).

8. Young NM. Structure of the $N$-Linked glycan present on multiple glycoproteins in the Gram-negative bacterium, Campylobacter jejuni. J. Biol. Chem. 277(45), 42530-42539 (2002). ** Determination of the $N$-linked glycan structure as a diNAcBac-containing heptasaccharide present on multiple proteins by the $N$-linked general glycosylation pathway in $C$. jejuni.

9. Mcnally DJ, Hui JPM, Aubry AJ et al. Functional characterization of the flagellar glycosylation locus in Campylobacter jejuni 81-176 using a focused metabolomics approach. J. Biol. Chem. 281(27), 18489-18498 (2006).

10. Ewing CP, Andreishcheva E, Guerry P. Functional characterization of flagellin glycosylation in Campylobacter jejuni 81-176. J. Bacteriol. 191(22), 7086-7093 (2009).

11. Doig P, Kinsella N, Guerry P, Trust TJ. Characterization of a post-translational modification of Campylobacter flagellin: Identification of a sero-specific glycosyl moiety. Mol. Microbiol. 19(2), 379-387 (1996).

12. Schirm M, Schoenhofen IC, Logan SM, Waldron KC, Thibault P. Identification of unusual bacterial glycosylation by tandem mass spectrometry analyses of intact proteins. Anal. Chem. 77(23), 7774-7782 (2005).

13. Schirm M, Soo EC, Aubry AJ, Austin J, Thibault P, Logan SM. Structural, genetic and functional characterization of the flagellin glycosylation process in Helicobacter pylori. Mol. Microbiol. 48(6), 1579-1592 (2003). * Demonsration of both FlaA and FlaB proteins are glycosylated with Pse in 


\section{H. pylori.}

14. Schoenhofen IC, Mcnally DJ, Brisson JR, Logan SM. Elucidation of the CMP-pseudaminic acid pathway in Helicobacter pylori: synthesis from UDP-N-acetylglucosamine by a single enzymatic reaction. Glycobiology 16(9), 8C-14C (2006). * Elucidation of biosynthetic pathway of sugar donor CMP-Pse utilized for flagellin glycosylation in $H$. pylori and $C$. jejuni.

15. Parker JL, Day-Williams MJ, Tomas JM, Stafford GP, Shaw JG. Identification of a putative glycosyltransferase responsible for the transfer of pseudaminic acid onto the polar flagellin of Aeromonas caviae Sch3N. Microbiologyopen 1(2), 149-160 (2012).

16. Schirm M, Arora SK, Verma A et al. Structural and genetic characterization of glycosylation of type a flagellin in Pseudomonas aemginosa. J. Bacteriol. 186(9), 2523-2531 (2004).

17. Schirm M, Kalmokoff M, Aubry A, Thibault P, Sandoz M, Logan SM. Flagellin from Listeria monocytogenes is glycosylated with beta-O-Linked $N$-acetylglucosamine. J. Bacteriol. 186(20), 6721-6727 (2004).

18. Shen A, Kamp HD, Grundling A, Higgins DE. A bifunctional $O$-GlcNAc transferase governs flagellar motility through anti-repression. Genes Dev. 20(23), 3283-3295 (2006).

19. Twine SM, Reid CW, Aubry A et al. Motility and flagellar glycosylation in Clostridium difficile. J. Bacteriol. 191(22), 7050-7062 (2009).

20. Power PM, Seib KL, Jennings MP. Pilin glycosylation in Neisseria meningitidis occurs by a similar pathway to wzy-dependent O-antigen biosynthesis in Escherichia coli. Biochem. Biophys. Res. Commun. 347(4), 904-908 (2006).

21. Vik A, Aas FE, Anonsen $\mathrm{JH}$ et al. Broad spectrum $O$-linked protein glycosylation in the human pathogen Neisseria gonorrhoeae. Proc Natl Acad Sci U S A 106(11), 4447-4452 (2009).

22. Faridmoayer A, Fentabil MA, Mills DC, Klassen JS, Feldman MF. Functional characterization of bacterial oligosaccharyltransferases involved in $O$-Linked protein glycosylation. J. Bacteriol. 189(22), 8088-8098 (2007).

23. Sherlock O, Dobrindt U, Jensen JB, Munk Vejborg R, Klemm P. Glycosylation of the self-recognizing Escherichia coli Ag43 autotransporter protein. $J$. Bacteriol. 188(5), 1798-1807 (2006).

24. Moormann C. Functional substitution of the TibC protein of enterotoxigenic Escherichia coli strains for the autotransporter adhesin heptosyltransferase of the AIDA system. Infect. Immun. 70(5), 2264-2270 (2002).

25. Charbonneau ME, Mourez M. The Escherichia coli AIDA-I autotransporter undergoes cytoplasmic glycosylation independently of export. Res. Microbiol. 159(7-8), 537-544 (2008).

26. Benz I, Schmidt MA. Glycosylation with heptose residues mediated by the aah gene product is essential for adherence of the AIDA-I adhesin. Mol. Microbiol. 40(6), 1403-1413 (2001).

27. Fletcher CM, Coyne MJ, Villa OF, Chatzidaki-Livanis M, Comstock LE. A 
General $O$-glycosylation system important to the physiology of a major human intestinal symbiont. Cell. 137(2), 321-331 (2009). ** Demonstration of a general OST-mediated protein $\boldsymbol{O}$-glycosylation systems in Bacteroides.

28. Zhang $\mathrm{H}$, Zhu F, Yang $\mathrm{T}$ et al. The highly conserved domain of unknown function 1792 has a distinct glycosyltransferase fold. Nat Commun. 5 (2014).

29. Grass S, Buscher AZ, Swords WE et al. The Haemophilus influenzae HMW1 adhesin is glycosylated in a process that requires HMW1C and phosphoglucomutase, an enzyme involved in lipooligosaccharide biosynthesis. Mol. Microbiol. 48(3), 737-751 (2003). * Identification of HMW1C as the glycosyltransferase required for the $\mathrm{N}$-linked glycosylation of HMW1 adhesion in $\mathrm{H}$. influenzae.

30. Grass S, Lichti CF, Townsend RR, Gross J, St Geme JW, Iii. The Haemophilus influenzae HMW1C protein is a glycosyltransferase that transfers hexose residues to asparagine sites in the HMW1 Adhesin. PLoS Pathog. 6(5), (2010).

31. Zeituni AE, Mccaig W, Scisci E, Thanassi DG, Cutler CW. The native 67-kilodalton minor fimbria of Porphyromonas gingivalis is a novel glycoprotein with DC-SIGN-targeting Motifs. J. Bacteriol. 192(16), 4103-4110 (2010).

32. Murakami Y, Hasegawa Y, Nagano K, Yoshimura F. Characterization of wheat germ agglutinin lectin-reactive glycosylated OmpA-like proteins derived from Porphyromonas gingivalis. Infect. Immun. 82(11), 4563-4571 (2014).

33. Gebhart C, Ielmini MV, Reiz B et al. Characterization of exogenous bacterial oligosaccharyltransferases in Escherichia coli reveals the potential for $O$-linked protein glycosylation in Vibrio cholerae and Burkholderia thailandensis. Glycobiology. 22(7), 962-974 (2012).

34. Scott AE, Twine SM, Fulton KM et al. Flagellar glycosylation in Burkholderia pseudomallei and Burkholderia thailandensis. J. Bacteriol. 193(14), 3577-3587 (2011).

35. Balonova L, Mann BF, Cerveny L et al. Characterization of protein glycosylation in Francisella tularensis subsp. holarctica: identification of a novel glycosylated lipoprotein required for virulence. Mol Cell Proteomics. 11(7), M111.015016 (2012).

36. Egge-Jacobsen W, Salomonsson EN, Aas FE et al. $O$-linked glycosylation of the PilA pilin protein of Francisella tularensis: identification of the endogenous protein-targeting oligosaccharyltransferase and characterization of the native oligosaccharide. J. Bacteriol. 193(19), 5487-5497 (2011).

37. Iwashkiw JA, Seper A, Weber BS et al. Identification of a general $O$-linked protein glycosylation system in Acinetobacter baumannii and its role in virulence and biofilm formation. PLoS Pathog. 8(6), (2012). * Demonstration of Acinetobacter baumannii possessing a general $O$-glycosylation system responsible for the glycosylation of multiple proteins.

38. Harding CM, Nasr MA, Kinsella RL et al. Acinetobacter strains carry two functional oligosaccharyltransferases, one devoted exclusively to type IV pilin, and the other one dedicated to $O$-glycosylation of multiple proteins. Mol. 
Microbiol. 96(5), 1023-1041 (2015).

39. Nothaft $\mathrm{H}$, Scott NE, Vinogradov E et al. Diversity in the protein N-glycosylation pathways within the Campylobacter genus. Mol. Cell. Proteomics 11(11), 1203-1219 (2012).

40. Thibault P, Logan SM, Kelly JF et al. Identification of the carbohydrate moieties and glycosylation motifs in Campylobacter jejuni flagellin. J. Biol. Chem. 276(37), 34862-34870 (2001).

41. $\mathrm{Ku}$ SC, Schulz BL, Power PM, Jennings MP. The pilin $O$-glycosylation pathway of pathogenic Neisseria is a general system that glycosylates AniA, an outer membrane nitrite reductase. Biochem. Biophys. Res. Commun. 378(1), 84-89 (2009).

42. Marino K, Bones J, Kattla JJ, Rudd PM. A systematic approach to protein glycosylation analysis: a path through the maze. Nat. Chem. Biol. 6(10), 713-723 (2010).

43. Kowarik M, Young NM, Numao S et al. Definition of the bacterial $N$-glycosylation site consensus sequence. EMBO J. 25(9), 1957-1966 (2006).

44. Lommel M, Strahl S. Protein O-mannosylation: conserved from bacteria to humans. Glycobiology 19(8), 816-828 (2009).

45. Schoenhofen IC, Mcnally DJ, Vinogradov E et al. Functional characterization of dehydratase/aminotransferase pairs from Helicobacter and Campylobacter: enzymes distinguishing the pseudaminic acid and bacillosamine biosynthetic pathways. J. Biol. Chem. 281(2), 723-732 (2006).

46. Olivier NB, Chen MM, Behr JR, Imperiali B. In vitro biosynthesis of UDP-N,N '-diacetylbacillosamine by enzymes of the Campylobacter jejuni general protein glycosylation system. Biochemistry. 45(45), 13659-13669 (2006).

47. Glover KJ, Weerapana E, Chen MM, Imperiali B. Direct biochemical evidence for the utilization of UDP-bacillosamine by $\mathrm{PglC}$, an essential glycosyl-1-phosphate transferase in the Campylobacter jejuni $\mathrm{N}$-linked glycosylation pathway. Biochemistry.45(16), 5343-5350 (2006).

48. Kelly J, Jarrell H, Millar L et al. Biosynthesis of the $N$-Linked glycan in Campylobacter jejuni and addition onto protein through block transfer. $J$. Bacteriol. 188(7), 2427-2434 (2006).

49. Troutman JM, Imperiali B. Campylobacter jejuni $\mathrm{PglH}$ is a single active site processive polymerase that utilizes product inhibition to limit sequential glycosyl transfer reactions. Biochemistry. 48(12), 2807-2816 (2009).

50. Linton D, Dorrell N, Hitchen PG et al. Functional analysis of the Campylobacter jejuni $\mathrm{N}$-linked protein glycosylation pathway. Mol. Microbiol. 55(6), 1695-1703 (2005).

51. Jervis AJ, Butler JA, Lawson AJ, Langdon R, Wren BW, Linton D. Characterization of the structurally diverse $N$-linked glycans of Campylobacter species. J. Bacteriol. 194(9), 2355-2362 (2012).

52. Jervis AJ, Langdon R, Hitchen $\mathrm{P}$ et al. Characterization of $N$-linked protein glycosylation in Helicobacter pullorum. J. Bacteriol. 192(19), 5228-5236 
(2010).

53. Hartley MD, Morrison MJ, Aas FE, Borud B, Koomey M, Imperiali B. Biochemical characterization of the $O$-linked glycosylation pathway in Neisseria gonorrhoeae responsible for biosynthesis of protein glycans containing N,N'-diacetylbacillosamine. Biochemistry. 50(22), 4936-4948 (2011).

54. Power PM, Roddam LF, Rutter K, Fitzpatrick SZ, Srikhanta YN, Jennings MP. Genetic characterization of pilin glycosylation and phase variation in Neisseria meningitidis. Mol. Microbiol. 49(3), 833-847 (2004).

55. Musumeci MA, Hug I, Scott NE et al. In vitro activity of Neisseria meningitidis PglL $O$-oligosaccharyltransferase with diverse synthetic lipid donors and a UDP-activated sugar. J. Biol. Chem. 288(15), 10578-10587 (2013).

56. Gault J, Ferber M, Machata S et al. Neisseria meningitidis Type IV pili composed of sequence invariable pilins are masked by multisite glycosylation. PLoS Pathog. 11(9), e1005162 (2015).

57. Hug I, Feldman MF. Analogies and homologies in lipopolysaccharide and glycoprotein biosynthesis in bacteria. Glycobiology. 21(2), 138-151 (2010).

58. Castric P. pilO, a gene required for glycosylation of Pseudomonas aeruginosa 1244 pilin. Microbiology. 141 ( Pt 5) 1247-1254 (1995).

59. Castric P, Cassels FJ, Carlson RW. Structural characterization of the Pseudomonas aeruginosa 1244 pilin glycan. J. Biol. Chem. 276(28), 26479-26485 (2001).

60. Digiandomenico A, Matewish MJ, Bisaillon A, Stehle JR, Lam JS, Castric P. Glycosylation of Pseudomonas aeruginosa 1244 pilin: glycan substrate specificity. Mol. Microbiol. 46(2), 519-530 (2002).

61. King JD, Kocincova D, Westman EL, Lam JS. Review: Lipopolysaccharide biosynthesis in Pseudomonas aeruginosa. Innate Immun. 15(5), 261-312 (2009).

62. Lees-Miller RG, Iwashkiw JA, Scott NE et al. A common pathway for $O$-linked protein-glycosylation and synthesis of capsule in Acinetobacter baumannii. Mol. Microbiol. 89(5), 816-830 (2013).

63. Posch G, Pabst M, Neumann L et al. "Cross-glycosylation" of proteins in Bacteroidales species. Glycobiology. 23(5), 568-577 (2013).

64. Thomas RM, Twine SM, Fulton KM et al. Glycosylation of DsbA in Francisella tularensis subsp. tularensis. J. Bacteriol. 193(19), 5498-5509 (2011).

65. Dankova V, Balonova L, Link M, Straskova A, Sheshko V, Stulik J. Inactivation of Francisella tularensis gene encoding putative ABC transporter has a pleiotropic effect upon production of various glycoconjugates. $J$. Proteome Res. 15(2), 510-524 (2016).

66. Twine SM, Vinogradov E, Lindgren H, Sjostedt A, Conlan JW. Roles for wbtC, wbtI, and kdtA genes in lipopolysaccharide biosynthesis, protein glycosylation, virulence, and immunogenicity in Francisella tularensis strain SCHU S4. 
Pathogens. 1(1), 12-29 (2012).

67. Dobos Km KK, Swiderek Km, Brennan Pj, Belisle Jt. Definition of the full extent of glycosylation of the 45-kilodalton glycoprotein of Mycobacterium tuberculosis. J. Bacteriol. 178(9), 2498-2506 (1996).

68. Liu CF, Tonini L, Malaga $\mathrm{W}$ et al. Bacterial protein- $O$-mannosylating enzyme is crucial for virulence of Mycobacterium tuberculosis. Proc Natl Acad Sci U S A 110(16), 6560-6565 (2013).

69. Mahne M, Tauch A, Puhler A, Kalinowski J. The Corynebacterium glutamicum gene pmt encoding a glycosyltransferase related to eukaryotic protein- $O$-mannosyltransferases is essential for glycosylation of the resuscitation promoting factor $(\mathrm{Rpf} 2)$ and other secreted proteins. FEMS Microbiol. Lett. 259(2), 226-233 (2006).

70. Wehmeier S, Varghese AS, Gurcha SS et al. Glycosylation of the phosphate binding protein, PstS, in Streptomyces coelicolor by a pathway that resembles protein O-mannosylation in eukaryotes. Mol. Microbiol. 71(2), 421-433 (2009).

71. Kawai F, Grass S, Kim Y, Choi KJ, St Geme JW 3rd, Yeo HJ. Structural insights into the glycosyltransferase activity of the Actinobacillus pleuropneumoniae HMW1C-like protein. J. Biol. Chem. 286(44), 38546-38557 (2011).

72. Cantarel BL, Coutinho PM, Rancurel C, Bernard T, Lombard V, Henrissat B. The Carbohydrate-Active EnZymes database (CAZy): an expert resource for Glycogenomics. Nucleic Acids Res. 37 D233-D238 (2009).

73. Choi KJ, Grass S, Paek S, St. Geme JW 3rd, Yeo HJ. The Actinobacillus pleuropneumoniae HMW1C-Like glycosyltransferase mediates $N$-Linked glycosylation of the Haemophilus influenzae HMW1 adhesin. PLoS One. 5(12), (2010).

74. Tytgat HL, Lebeer S. The sweet tooth of bacteria: common themes in bacterial glycoconjugates. Microbiol. Mol. Biol. Rev. 78(3), 372-417 (2014).

75. Logan SM. Flagellar glycosylation - a new component of the motility repertoire? Microbiology. 152(5), 1249-1262 (2006).

76. Mcnally DJ, Aubry AJ, Hui JP et al. Targeted metabolomics analysis of Campylobacter coli VC167 reveals legionaminic acid derivatives as novel flagellar glycans. J. Biol. Chem. 282(19), 14463-14475 (2007).

77. Josenhans C, Vossebein L, Friedrich S, Suerbaum S. The neuA/flmD gene cluster of Helicobacter pylori is involved in flagellar biosynthesis and flagellin glycosylation. FEMS Microbiol. Lett. 210(2), 165-172 (2002).

78. Schoenhofen IC. Structural and functional characterization of PseC, an aminotransferase involved in the biosynthesis of pseudaminic acid, an essential flagellar modification in Helicobacter pylori. J. Biol. Chem. 281(13), 8907-8916 (2006).

79. Schoenhofen IC, Vinogradov E, Whitfield DM, Brisson J-R, Logan SM. The CMP-legionaminic acid pathway in Campylobacter: biosynthesis involving novel GDP-linked precursors. Glycobiology. 19(7), 715-725 (2009). * 
Elucidation of biosynthetic pathway of sugar donor CMP-Leg utilized for flagellin glycosylation in $\boldsymbol{C}$. jejuni.

80. Twine SM, Paul CJ, Vinogradov E et al. Flagellar glycosylation in Clostridium botulinum. FEBS J. 275(17), 4428-4444 (2008).

81. Celik N, Webb CT, Leyton DL et al. A bioinformatic strategy for the detection, classification and analysis of bacterial autotransporters. PLoS One. 7(8), e43245 (2012).

82. Cote JP, Charbonneau ME, Mourez M. Glycosylation of the Escherichia coli TibA self-associating autotransporter influences the conformation and the functionality of the protein. PLoS One. 8(11), e80739 (2013).

83. Sherlock O, Vejborg RM, Klemm P. The TibA adhesin/invasin from enterotoxigenic Escherichia coli is self recognizing and induces bacterial aggregation and biofilm formation. Infect. Immun. 73(4), 1954-1963 (2005).

84. Van Der Woude MW, Henderson IR. Regulation and function of Ag43 (flu). Annu. Rev. Microbiol. 62 153-169 (2008).

85. Hug I, Couturier MR, Rooker MM, Taylor DE, Stein M, Feldman MF. Helicobacter pylori lipopolysaccharide is synthesized via a novel pathway with an evolutionary connection to protein $\mathrm{N}$-glycosylation. PLoS Pathog. 6(3), e1000819 (2010).

86. Wacker M, Linton D, Hitchen PG et al. $N$-linked glycosylation in Campylobacter jejuni and its functional transfer into E. coli. Science 298(5599), 1790-1793 (2002).

87. Wacker M. Substrate specificity of bacterial oligosaccharyltransferase suggests a common transfer mechanism for the bacterial and eukaryotic systems. Proc Nati Acad Sci U S A. 103(18), 7088-7093 (2006). ** Demonstration of the use of bacteria for glycoengineering a number of recombinant proteins.

88. Feldman MF. Engineering $N$-linked protein glycosylation with diverse $\mathrm{O}$ antigen lipopolysaccharide structures in Escherichia coli. Proc Nati Acad Sci US A. 102(8), 3016-3021 (2005).

89. Faridmoayer A, Fentabil MA, Haurat MF et al. Extreme substrate promiscuity of the neisseria oligosaccharyl transferase involved in protein $O$-glycosylation. J. Biol. Chem. 283(50), 34596-34604 (2008). ** Demonstration of the OST PglL from $N$. meningitidis is able to transfer virtually any glycan including the $C$. jejuni N-linked glycan, the LPS O-antigen and peptidoglycan subunits to pilin in engineered $E$. coli and Salmonella cells.

90. Hug I, Zheng B, Reiz B et al. Exploiting bacterial glycosylation machineries for the synthesis of a Lewis antigen-containing glycoprotein. J. Biol. Chem. 286(43), 37887-37894 (2011).

91. Lizak C, Gerber S, Numao S, Aebi M, Locher KP. X-ray structure of a bacterial oligosaccharyltransferase. Nature. 474(7351), 350-355 (2011). 


\section{Figure Legends}

\section{Figure 1. Lipid Carrier-Mediated en bloc Protein Glycosylation Pathways}

A. En bloc protein $N$-glycosylation pathway in $C$. jejuni. Three enzymes PglF, PglE and PgID are responsible for the conversion of UDP-GlcNAc to UDP-diNAcBac, which is the first sugar residue transferred to UndP carrier by PglC to form UndPP-linked diNAcBac. Further actions of GTs PglA, PglJ and PglH add five GalNAc residues and one branched Glc residue to complete the UndPP-linked heptosaccharide. Once completed, the LLO is flipped by the flippase PglK into the periplasm, where the OST PglB transfers the glycan to the Asn residue present in the protein consensus sequence:

Asp/Glc-X1-Asn-X2-Ser/Thr(D/E-X1-N-X2-S/T) (X1 and X2 represent any amino acid except proline).

B. En bloc protein $O$-glycosylation pathway in $N$. meningitidis and $N$. gonorrhoeae. The sequential actions of $\mathrm{PglD}, \mathrm{PglC}$, and $\mathrm{PglB}$ or PglB2 are involved in the conversion of UDP-GlcNAc to UDP-DATDH (by PglB) or UDP-GATDH (by PglB2). The bifunctional PglB and PglB2 also function to transfer DATDH and GATDH to the lipid carrier, respectively. Further actions of GTs PglA and PglE add Gal residues to complete the UndPP-linked trisaccharide. The LLO is then flipped by the flippase PglF into the periplasm, where the OST PglL in $N$. meningitidis or the OST PglO in N. gonorrhoeae transfers the glycan to Ser/Thr $(\mathrm{S} / \mathrm{T})$ residues of target proteins.

C. En bloc protein $O$-glycosylation pathway in $P$. aeruginosa 1244 . It is proposed that $\mathrm{WbpM}$ and $\mathrm{WbpK}$ are responsible for the conversion of UDP-GlcNAc to UDP-FucNAc, which is the first sugar transferred to the lipid carrier by WbpL. Further actions of unknown GTs add Xyl and $\alpha-5 \mathrm{~N} \beta \mathrm{OHC} 47 \mathrm{NFmPse}$ residues to complete the UndPP-linked trisaccharide. The LLO is then flipped by the O-antigen flippase Wzx into the periplasm, where the OST PilO transfers the glycan to the Ser/Thr $(\mathrm{S} / \mathrm{T})$ residues of PilA.

D. En bloc protein $O$-glycosylation pathway in A. baumannii ATCC 17978. It is proposed that PglC transfers the first sugar GalNAc to the lipid carrier. Further actions of four putative GTs (A1S_0058, A1S_3482, A1S_0059, and A1S_0060) add Glc, GlcNAc, Gal and UDP-GlcNAc3NAcA4OAc to complete the UndPP-linked pentasaccharide. The LLO is then flipped by the $O$-antigen flippase Wzx (A1S_0056) into the periplasm, where the OST PgIL (A1S_3176) transfers the glycan to the Ser/Thr $(\mathrm{S} / \mathrm{T})$ residues of target proteins. The biosynthesis of UDP-GlcNAc3NAcA4OAc from UDP-GlcNAc is proposed to be 
catalyzed by several enzymes (A1S_0052, A1S_0053, A1S_0055, A1S_0054 and A1S_0057).

\section{Figure 2. The Biosynthetic Pathway for UDP-diNAcBac in C. jejuni}

The biosynthesis of UDP-diNAcBac involves three steps starting from UDP-GlcNAc.

Step 1: the conversion of UDP-GlcNAc to

UDP-2-acetamido-2,6-dideoxy- $\alpha$-D-xylo-4-hexulose by dehydratase PglF (Cj1120c); Step 2: the transfer of an amino group by aminotransferase PglE (Cj1121c), forming UDP-4-amino-4,6-dideoxy- $\alpha$-D-GlcNAc; Step 3 is catalyzed by acetyl transferase $\operatorname{PglD}(\mathrm{Cj} 1123 \mathrm{c})$.

Figure 3. The Biosynthetic Pathway for UDP-DATDH and UDP-GATDH in $N$. meningitidis and $N$. gonorrhoeae.

The biosynthesis of UDP-DATDH and UDP-GATDH involves three steps starting from UDP-GlcNAc. Step 1 and Step 2 are the same as the first two steps of the biosynthesis of UDP-diNAcBac in C. jejuni (Figure 2), by PgID (homologous to the C. jejuni PglF) and $\mathrm{PglC}$ (homologous to the C. jejuni PglE), respectively; Step 3 is catalyzed by the acetyltransferase function of PglB or PglB2 to form UDP-DATDH or UDP-GATDH, respectively.*the structure of DATDH has been shown to be diNAcBac [53].

\section{Figure 4. Stepwise Protein Glycosylation Pathways}

A. Stepwise protein $N$-glycosylation pathway in $H$. influenzae. The nucleotide activated sugars UDP-Gal and UDP-Glc are individually transferred to the eukaryotic-like sequon: Asn-X-Ser/Thr of HMW1 by the GT HMW1C.

B. Stepwise protein $O$-glycosylation of flagellins in $C$. jejuni. The nucleotide activated sugars CMP-Pse and CMP-Leg, and their derivatives are sequentially added to the serine or threonine residues of FlaA of $C$. jejuni.

C. Stepwise protein $O$-glycosylation of flagellins in $\mathrm{H}$. pylori. The nucleotide activated sugar CMP-Pse is individually transferred to the serine or threonine residues of FlaA and FlaB of $H$. pylori.

\section{Figure 5. The Biosynthetic Pathway for CMP-Pse in $\mathrm{H}$. pylori}

The biosynthesis of CMP-Pse involves six steps starting from UDP-GlcNAc. Step 1: the dehydration and epimerization of GlcNAc by the bi-functional 4,6-dehydratase/5-epimerase PseB (HP0840), forming UDP-2-acetamido-2,6-dideoxy- $\beta$-L-arabino-hexos-4-ulose; Step 2: the aminotransfer by the aminotransferase PseC (HP0366), forming 
UDP-4-amino-4,6-dideoxy- $N$-acetyl- $\beta$-L-altrosamine; Step 3: the aceylation by the $N$-acetyltransferase PseH(HP0327),

forming UDP-2,4-bis(acetamido)-2,4,6-trideoxy- $\beta$-L-altropyranose; Step 4: the removal of UDP by the nucleotidase PseG (HP0326B); Step 5: the synthesis of Pse by the Pse synthetase PseI (HP0178); Step 6: the production of CMP-Pse by the CMP-Pse synthetase PseF (HP0326A).

\section{Figure 6. The Biosynthetic Pathway for CMP-Leg in C. jejuni}

The biosynthesis of CMP-Leg involves ten steps starting from Fru-6P (I). Step 1: Fru-6P (I) is converted to GlcN-6P (II) by the actions of the glutaminase PtmA $(\mathrm{Cj} 1332)$ and the isomerase PtmF (Cj1330); Step 2: the conversion of GlcN-6P to GlcN-1P (III) by the mutase PgmL(Cj1407c); Step 3: the formation of GDP-GlcN (IV) by the guanylytransferase PtmE (Cj1329); Step 4: the formation of GDP-GlcNAc (V) by the action of $N$-acetyltransferase GlmU (Cj0821); Step 5: the dehydration of V by the dehydratase $\quad \operatorname{LegB}(\mathrm{Cj} 1319), \quad$ forming GDP-2-acetamido-2,6-dideoxy- $\alpha$-D-xylo-hexos-4-ulose (VI); Step 6 : the aminotransfer of VI by the aminotransferase LegC (Cj1320), forming GDP-4-amino-4,6-dideoxy- $\alpha$-D-GlcNAc (VII) ; Step 7: the $N$-acetyltransfer of VII by the $N$-acetyltransferase LegH forming GDP-2,4-diacetamido-2,4,6-trideoxy- $\alpha$-D-glucopyranose (VIII) ; Step 8: the removal of NDP from VIII by the NDP-sugar hydrolase/2-epimerase LegG (Cj1328), forming 2,4-diacetamido-2,4,6-trideoxy-D-mannopyranose (IX); Step 9: the condensation of IX with pyruvate by the Leg synthase LegI (Cj1327), forming Leg (X); Step 10: the CMP-activation of Leg by the CMP-Leg synthetase LegF (Cj1331), producing CMP-Leg (XI).

\section{Figure 7. Stepwise Heptosylation of Autotransporters}

A. The genomic organization of autotransporters and their corresponding BAHTs in three representative bacterial strains. The gene encoding the BAHT is always located upstream of its substrate autotransporter in the same operon of the genome.

B. The biosynthetic pathway for ADP-LD-Hep and ADP-DD-Hep in Gram-negative bacteria. Four steps are involved for the production of ADP-DD-Hep starting from D-sedo-heptulose-7P. Step 1: the conversion to DD-Hep-7P by isomerase GmhA; Step 2: the conversion to DD-Hep-1,7PP by the bi-functional RfaE (kinase/adenosyltransferase); Step 3: the conversion to DD-Hep-1P by phosphatase GmhB; Step 4: the production of ADP-DD-Hep by the bi-functional RfaE (kinase/adenosyltransferase). For the production of ADP-LD-Hep, it is 
converted from ADP-DD-Hep by epimerase RfaD.

C. The pathway for the stepwise heptosylation of autotransporters. Prior to its secretion, the autotransporter is heptosylated by the heptosyltransferase BAHT on numerous Ser residues in the passenger domain. Both ADP-DD-Hep and ADP-LD-Hep derived from LPS biosynthetic pathway can serve as the sugar donors for the heptosylation of autotransporter by BAHT. 


\section{Figures}

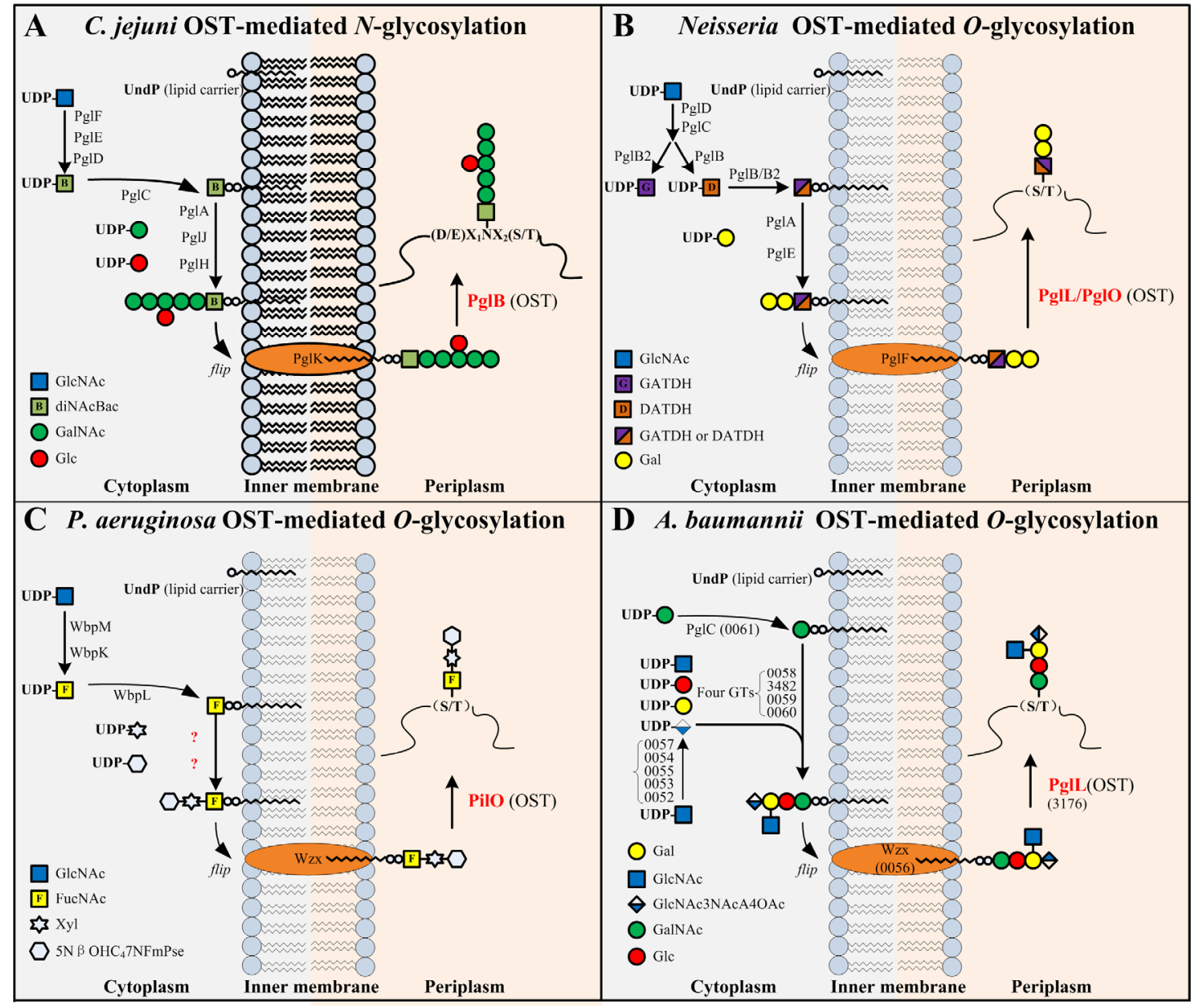

Figure 1 


\section{UDP-GlcNAc}

$$
\downarrow \operatorname{PglF}(\mathrm{Cj} 1120 \mathrm{c})
$$

UDP-2-acetamido-2,6-dideoxy- $\alpha$-D-xylo-4-hexulose

$$
\downarrow \text { PglE (Cj1121c) }
$$

UDP-4-amino-4,6-dideoxy- $\alpha$-D-GlcNAc

$$
\text { UDP-diNAcBac }
$$

Figure 2 


\section{UDP-GlcNAc}

$$
\downarrow^{\text {PgID }}
$$

UDP-2-acetamido-2,6-dideoxy- $\alpha$-D-xylo-4-hexulose

$$
\downarrow \text { PglC }
$$

UDP-4-amino-4,6-dideoxy- $\alpha$-D-GlcNAc

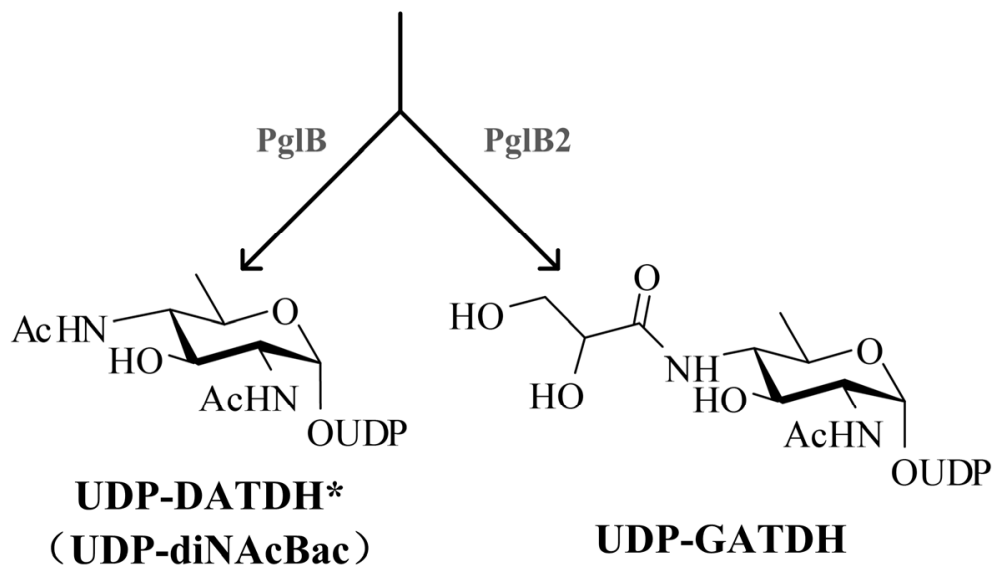

Figure 3 


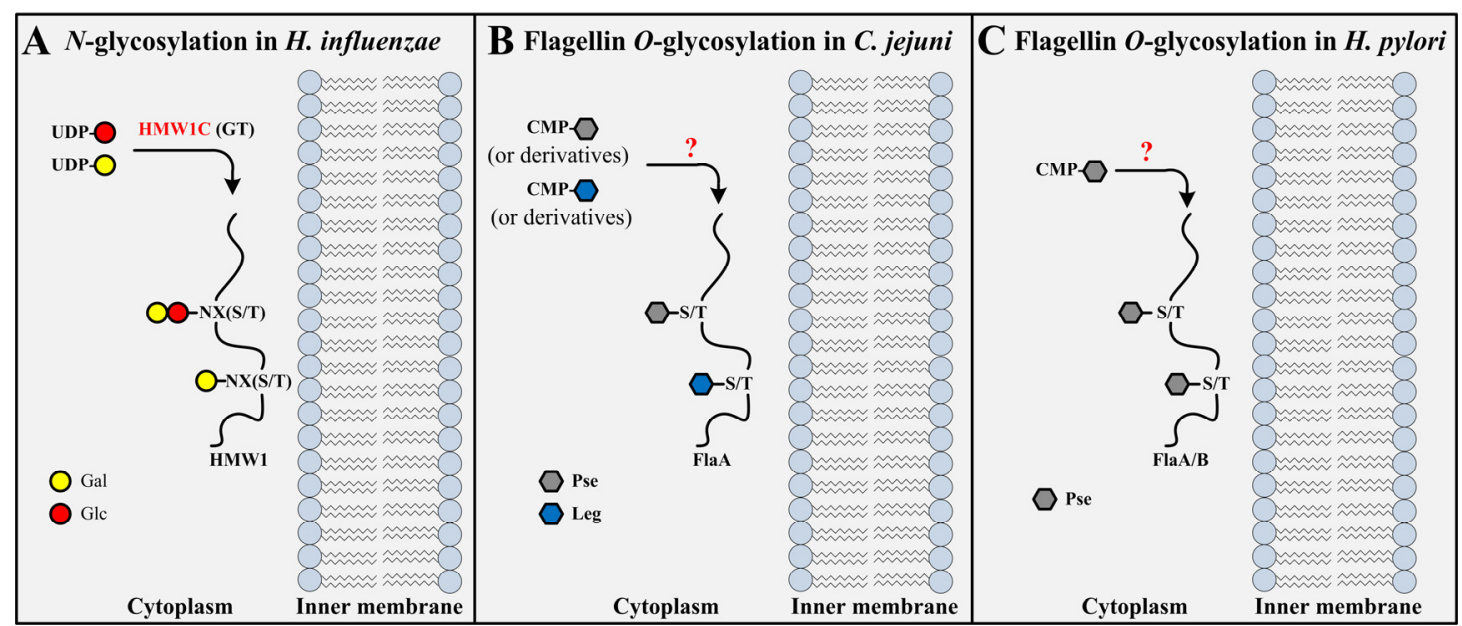

Figure 4 
UDP-GlcNAc

$$
\downarrow \text { PseB (HP0840) }
$$

UDP-2-acetamido-2,6-dideoxy- $\beta$-L-arabino-hexos-4-ulose

$$
\downarrow \text { PseC(HP0366) }
$$

UDP-4-amino-4,6-dideoxy- $N$-acetyl- $\beta$-L-altrosamine

$$
\downarrow \text { PseH (HP0327) }
$$

UDP-2,4-bis(acetamido)-2,4,6-trideoxy- $\beta$-L-altropyranose

$$
\downarrow \text { PseG (HP0326B) }
$$

2,4-bis(acetamido)-2,4,6-trideoxy- $\beta$-L-altropyranose

$$
\downarrow \text { PseI (HP0178) }
$$

Pse

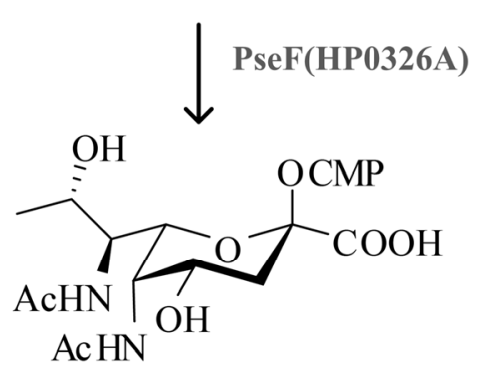

CMP-Pse

Figure 5 


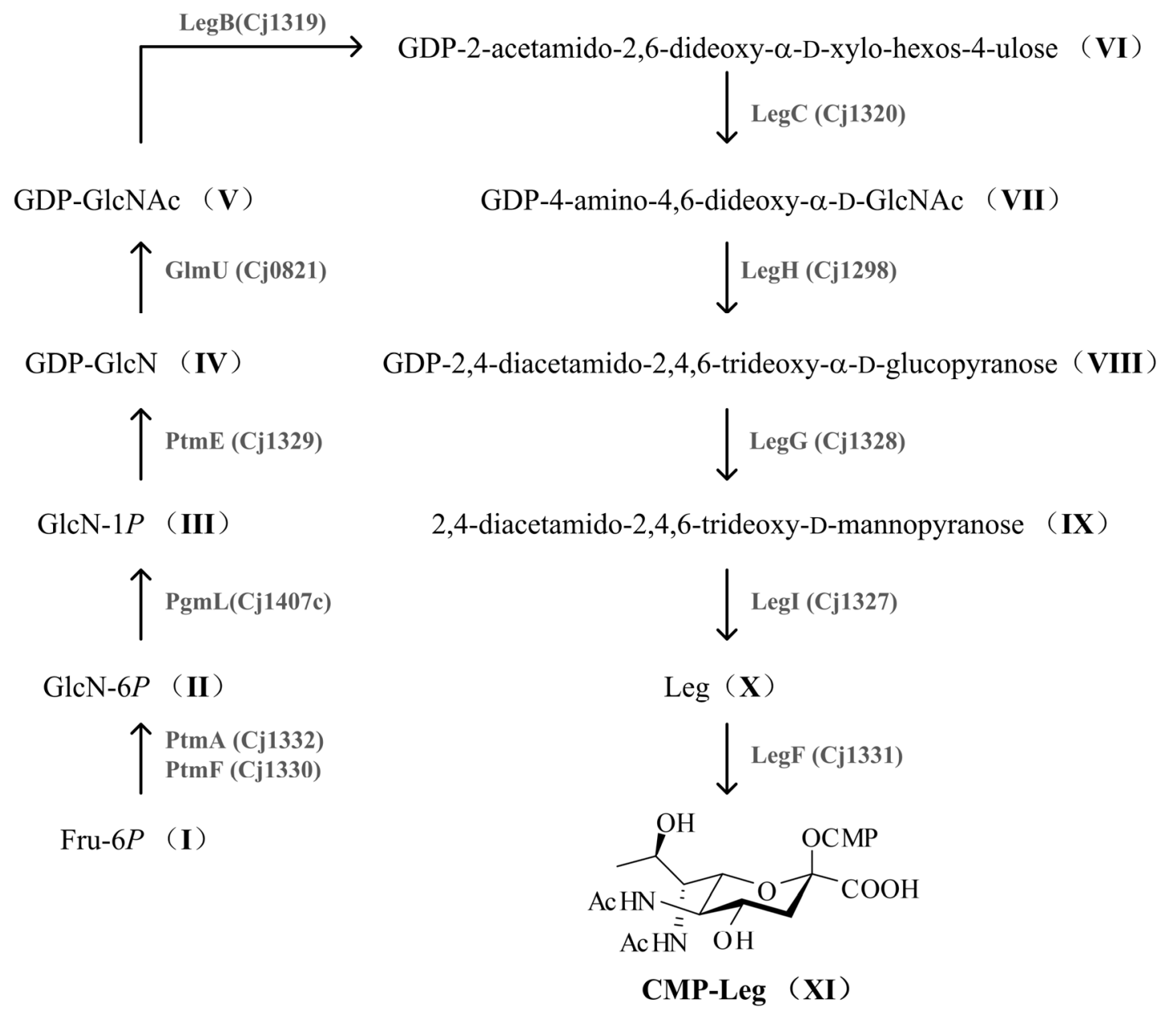

Figure 6 

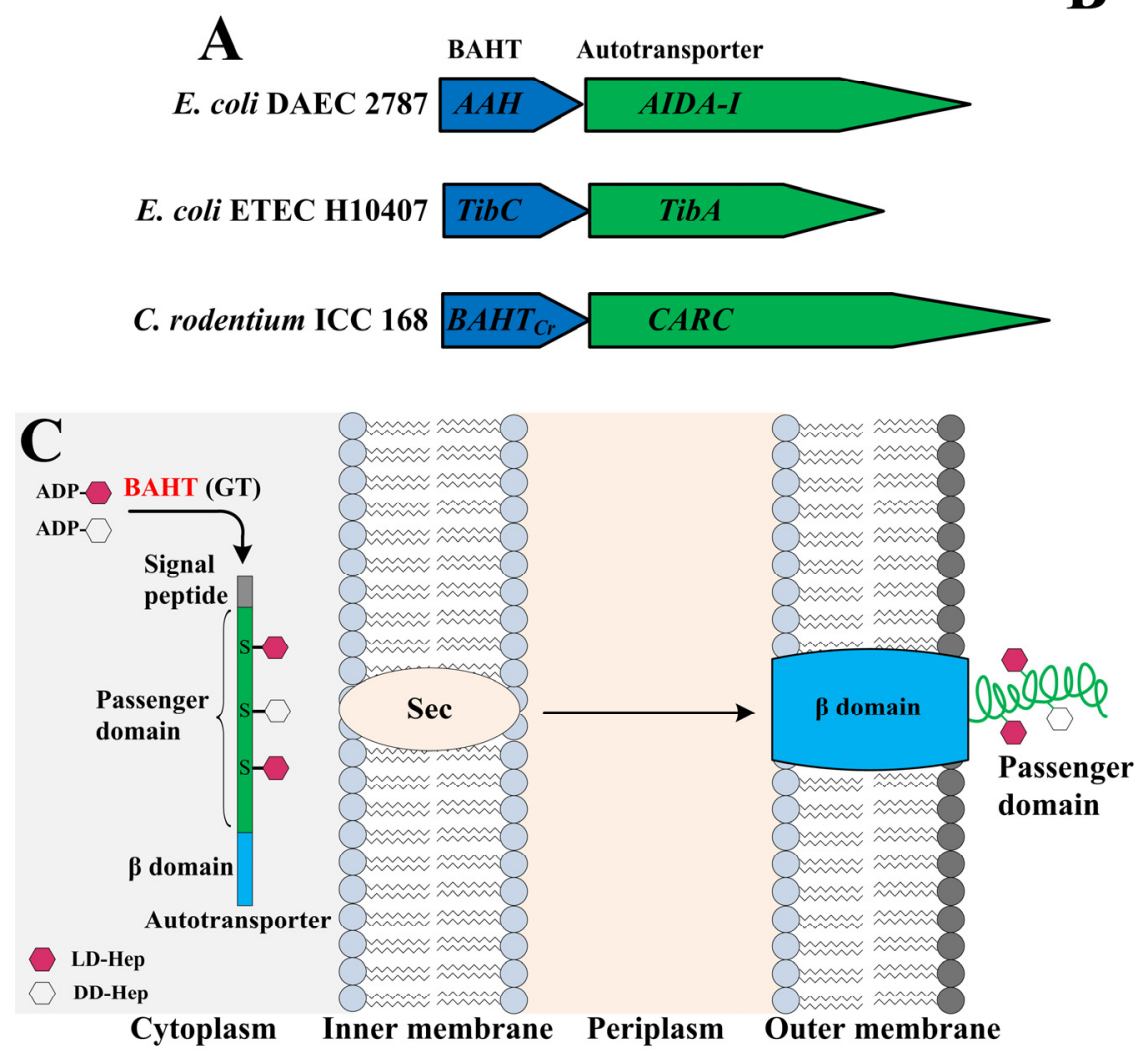

D-sedo-heptulose-7P

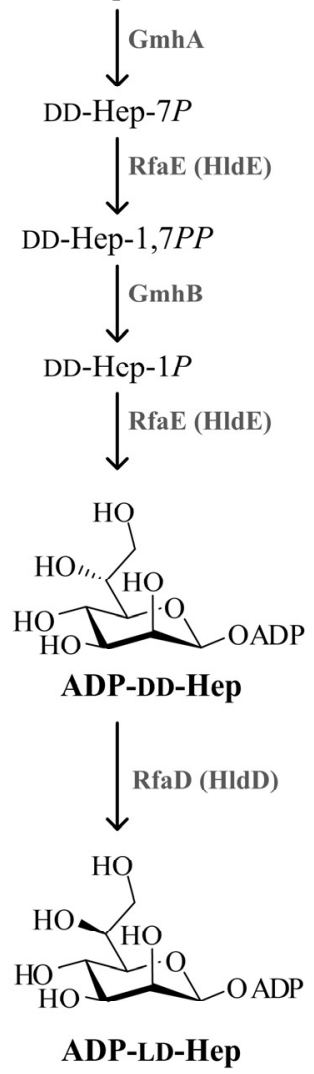

Figure 7 